\title{
Empirical Evidence of the Effectiveness of Concept Mapping as a Learning Intervention for Nuclear Medicine Technology Students in a Distance Learning Radiation Protection and Biology Course
}

\author{
Gregory G. Passmore, Mary Anne Owen, and Krishnan Prabakaran \\ Department of Medical Laboratory, Imaging, and Radiologic Sciences, Georgia Health Sciences University, Augusta, Georgia
}

\begin{abstract}
Metacognitive learning strategies are based on instructional learning theory, which promotes deep, meaningful learning. Educators in a baccalaureate-level nuclear medicine technology program demonstrated that students enrolled in an online, distance learning section of an introductory radiation protection and radiobiology course performed better when traditional instruction was supplemented with nontraditional metacognitive learning strategies. Methods: The metacognitive learning strategy that was used is best known as concept mapping. The concept map, in addition to the standard homework problem assignment and opportunity for question-answer sessions, became the template for misconception identification and remediation interactions between the instructor and the student. The control group relied on traditional homework problems and question-answer sessions alone. Because students in both the "treatment" groups (i.e., students who used concept mapping) and the control group were distance learning students, all personal communications were conducted via e-mail or telephone. The final examination of the course was used to facilitate a quantitative comparison of the performance of students who used concept mapping and the performance of students who did not use concept mapping. Results: The results demonstrated a significantly higher median final examination score for the concept mapping group than for the non-concept mapping group $(z=$ $-2.0381, P=0.0415)$, with an appropriately large effect size (2.65). Conclusion: Concept mapping is a cognitive learning intervention that effectively enables meaningful learning and is suitable for use in the independent learner-oriented distance learning environments used by some nuclear medicine technology programs.
\end{abstract}

\footnotetext{
Received May 11, 2011; revision accepted Aug. 9, 2011.

For correspondence or reprints contact: Gregory G. Passmore, Department of Medical Laboratory, Imaging, and Radiologic Sciences, Georgia Health Sciences University, 1120 15th St., Bldg. EC 2408, Augusta, GA 30912.

E-mail: gpassmor@georgiahealth.edu

Published online Nov. 11, 2011.

COPYRIGHT @ 2011 by the Society of Nuclear Medicine, Inc.
}

Key Words: concept map; distance learning; nuclear medicine technology; online education; radiation protection; radiobiology instruction

J Nucl Med Technol 2011; 39:284-289

DOI: $10.2967 /$ jnmt.111.093062

U enerally speaking, instruction exists on a continuum from teacher centered-content oriented to student centeredlearner oriented (1). Various models of this continuum exist in online instruction; they elicit reactive to proactive student behavior and participation and passive to active involvement of the instructor in learning. In a phenomenographic perspective of lecturers' experiences and philosophies of online teaching and student interactions (2), Gonzalez confirmed the findings of Kember and Kwan (3) that "teachers on the side of 'transmitting knowledge' were more likely to rely on 'content-centered' approaches ... while those who saw learning as a process of 'facilitating understanding' would rely more on 'learning-centered' methods." The curriculum for entry-level nuclear medicine technology instruction traditionally has been developed to meet the behavioral learning outcomes desired by national accrediting and credentialing bodies. Nuclear medicine technology instruction, whether through traditional classroom delivery or nontraditional online delivery, is, by design, "content centered" to meet these desired outcomes. However, it is our assertion that cognitivistor constructivist-based "learner-centered" methods of instruction are as effective as or more effective than traditional behaviorist-based content-centered methods and that these learner-centered methods can be successfully implemented through nontraditional online delivery systems.

In the baccalaureate-level nuclear medicine technology program at Georgia Health Sciences University, the required 5 -semester curriculum is $100 \%$ online and delivered in 
blended instructional formats. The program serves both traditional campus-based students and distance learning students who come to campus only occasionally for laboratory instruction.

The only instructional difference between students residing on campus and nonresident distance learning students is that students residing on campus have traditional expository classroom instruction in most junior-year courses. Distance learning students receive the same expository instruction asynchronously in the form of prerecorded lectures via the Tegrity lecture capture system (McGraw-Hill Higher Education). All courses require online interactions regardless of location. Instruction also includes various forms of cognitivist or constructivist learning interventions, such as "reflection questions" that involve online discussions by all students regardless of campus, group collaborative assignments that cross geographic locations and involve online exchanges of documents and ideas, online testing, self-paced completion of online assignments, and concept mapping to elicit a student's cognitive schema (allowing for misconception remediation) and to target deep understanding and meaningful learning of the abstract concepts in radiation protection and radiobiology.

This investigation was designed to measure the effectiveness of monitoring a student's conceptual schema and misconception remediation through the use of the cognitivist-based graphic organizer tool known as concept mapping in an introductory radiation protection and radiobiology course for distance learning students in a nuclear medicine technology program.

\section{Literature Review: How Do We Learn?}

Learning theories exist on a continuum. At the beginning is the behaviorist theory, in which learning is confirmed by specific behavioral responses to specific stimuli. Next is the cognitivist theory, in which learning is confirmed by behavioral and mental solutions to specific stimuli. At the end is the constructivist theory, in which learning is individualized and based on stimuli as they are presented. This continuum may be described as moving from rote learning or memorization, through meaningful learning and problem solving, to autonomous learning and invention $(1,4,5)$.

An example of the application of behaviorist theory to nuclear medicine technology is a student who correctly performs an imaging protocol in a clinic. This theory fails when the student is placed in a different clinic and is exposed to variations in the protocol. The student has been conditioned to respond to certain stimuli in the protocol, but when the learning stimuli change, the learner cannot appropriately respond unless he or she has memorized all variations of the protocol.

An example of the application of cognitivist theory to nuclear medicine technology is a student who has been given variations in clinical protocols. A student taught with cognitivist learning theory understands the need for the variations because this student has developed mental schema that allow for processing of problem-solving strategies that come with meaningful learning; therefore, this student does not view the required variations as an impediment to accomplishing the task.

An example of the application of constructivist theory to nuclear medicine technology is a student who has been given the task of inventing a protocol. Knowledge of previous protocols might be advantageous but might not be necessary. Constructivist learning supports creativity in problem solving because the student must approach the problem from different perspectives. A weakness of constructivist learning occurs when conformity is essential and divergent thinking might cause problems. By definition, a protocol establishes a set of procedures used to perform a task. Reinventing a set of procedures every time a task is required would be time-consuming and inefficient.

Instructional designers such as Ertner and Newby (1) assert that the learning theory that is to be used in the instruction should be the one that best meets the student's level of competence, the type of learning task, and the objectives of the instructional outcomes. The educators in the nuclear medicine technology program considered here believe that instruction that both meets the predicated curricular objectives of its governing agencies and promotes meaningful learning will best meet the needs of the student. The purpose of instruction, then, is for the student to develop a knowledge base within the discipline as well as to be able to learn new knowledge as the discipline changes with advancements in technology and practice. A student who is taught how to learn meaningfully through the nonarbitrary assimilation of new information or new concepts and procedures into a knowledge structure should be able to meet the discipline's needs now and in the future.

Educational theorists such as Novak and Gowen (6) believe that the key to meaningful learning is how a student organizes and retrieves information or knowledge. Meaningful learning is based on the premise that knowledge is bundled in packets called concepts and is retrievable on the basis of how the concepts are linked to one another. A concept is defined as a perceived regularity in an event or object designated by a label, such as a word or explicit symbol. Concept maps are graphic organizers of multiple concepts that represent an individual's knowledge of a topic or process (6-9). Two or more concepts and their linking relationships become meaningful statements about some object or event that an individual is trying to define or classify. The characteristic linking relationship of a concept map is what makes this graphic organizer unique and separates the concept map from other organizational techniques, such as outlines or flow charts. An important characteristic of concept maps is the inclusion of cross-links, which are relational links between concepts in different levels or conceptual clusters of the concept map. Cross-links help reveal how a concept in one domain of knowledge represented on the map is related to a concept in another domain shown on the map $(5-10)$. 
Meaningful learning requires a learner to become an active participant in the learning process. The process of generating a concept map by identifying relevant concepts and the relationships between them is an active learning, metacognitive process. Metacognitive strategies such as concept mapping allow a learner to actively organize a knowledge structure into a more powerful, integrated pattern (5,7-11). A concept map then becomes a concise, visual interpretation of an individual's knowledge structure; such a tool can simplify discussion and lead to enhanced understanding between individuals $(4,5,7-9,12)$. Figure 1 represents a student concept map depicting their understanding of fundamental radiation safety concepts by using relational linking terms between the concepts.

In a nuclear medicine technology curriculum, Passmore $(5,7,8)$ used concept maps effectively as a remediation tool, prompting communication between instructor and student and allowing for misconception identification and subsequent remediation activities. This interaction demonstrated an increased understanding of the radiologic science subject matter on the basis of the increased complexity of the student's concept maps. Concept maps have also been used to analyze teacher-student and/or group differences in the understanding of processes, strategies, and products; to observe differences in students' understanding of procedural systems, such as imaging protocols; and to assess specific knowledge of related processes so that learning and associated objectives can be achieved $(4,10,13)$.

\section{Research Question}

If nuclear medicine technology students who are enrolled in a distance learning program prepare concept maps as metacognitive strategies and use them as a basis for correspondence with their instructors, then will their final examination scores be higher than those of nuclear medicine technology students who are enrolled in a distance learning program but do not prepare concept maps as a basis for correspondence with their instructors?

\section{MATERIALS AND METHODS}

\section{Subjects}

The subjects for this study were nuclear medicine technology students enrolled in the radiation protection and radiobiology curriculum component of a distance learning nuclear medicine technology program between 2007 and 2010. The total number of students consenting to participate in the study was 25 . The learning intervention (concept mapping) group consisted of 19 students. The control

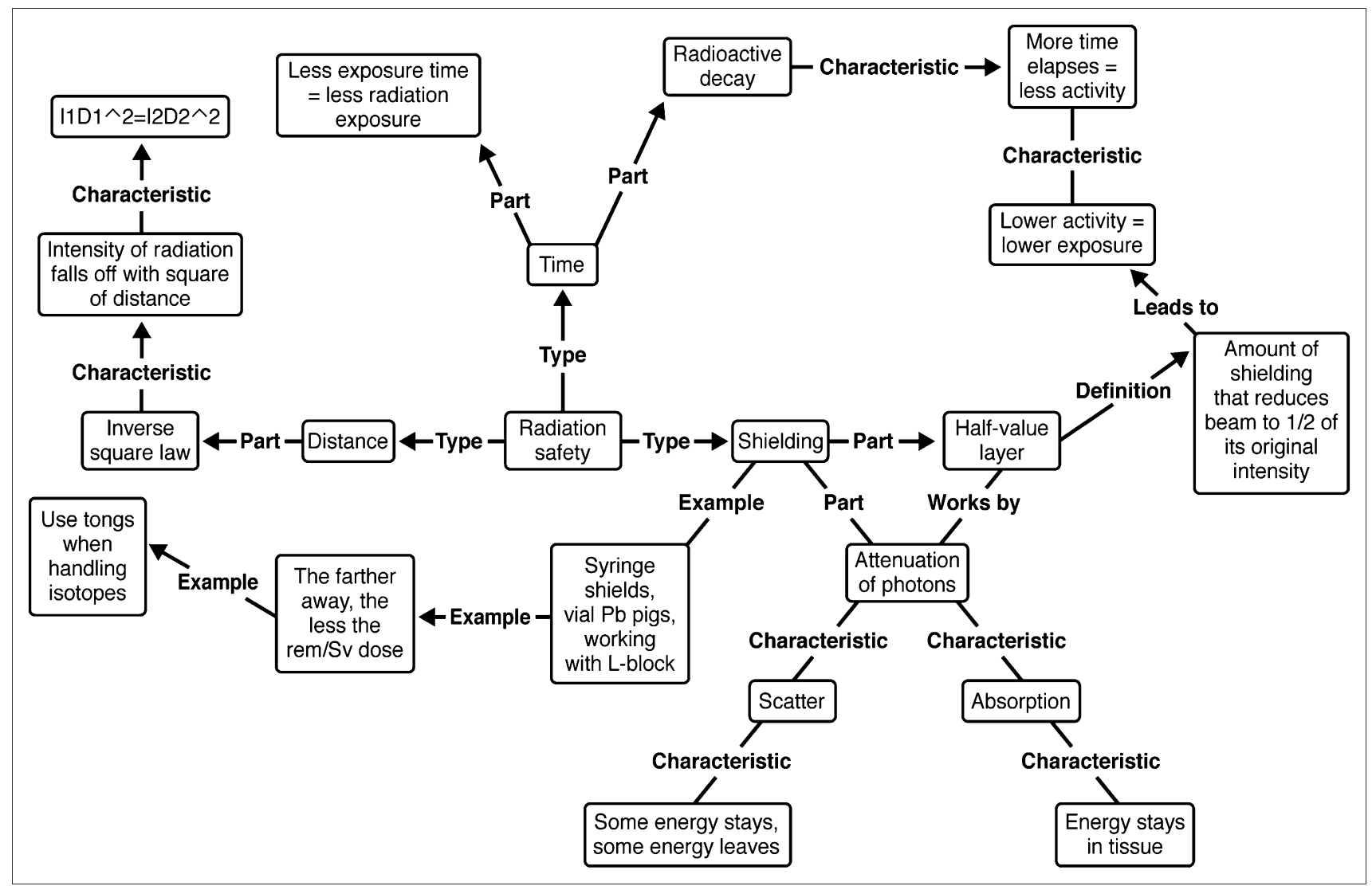

FIGURE 1. Student concept map depicting basic concepts of radiation protection: time, distance, and shielding. Note complexity and linking relationships between concepts and between clusters, indicating meaningful understanding of components of radiation protection and how they work together. 
(non-concept mapping) group consisted of 6 students. All students entering the nuclear medicine technology program must have a minimum grade point average of 2.5 in mathematics and science and a minimum overall grade point average of 2.3. Furthermore, all students entering the nuclear medicine technology program are interviewed before selection with a rating scale developed for the interview process. Neither SAT or ACT scores nor an entrance examination is used by the nuclear medicine technology program to establish qualification for entrance into the program.

The research was approved by the Human Assurance Committee of Georgia Health Sciences University.

\section{Design}

The overall design of this investigation is a quasiexperimental 2-group comparison. This design was implemented because of the condition of using intact classes as treatment and control groups. Groups designated by the years 2007, 2008, and 2009 were used as treatment groups until an appropriate sample size was achieved. The group designated by the year 2010 was used as a control group. Equivalence between the treatment groups and the control group was based on equivalent minimum entrance requirements (as described earlier), and no covariate analyses were considered in this research design.

The concept mapping group received $3 \mathrm{~h}$ of workshop instruction and practice on concept mapping during student orientation, before the start of didactic and clinical instruction during the fall semester, which covered an academic period of $16 \mathrm{wk}$.

Concept map summaries of chapters, laboratories, or application problems were requested from students before each of the 3 examinations given during the semester. Students could develop their concept maps by hand or by computer and could e-mail the maps to their instructors as part of the mandatory weekly correspondence with their instructors. Student misconceptions displayed in the concept maps were addressed by the instructor and the student. This interaction typically included questions directed at the student's map with the intent of helping the student identify misconceptions. This process was conducted by telephone, fax, or e-mail and was repeated as time allowed, until the student reached cognitive congruence with the instructor.

The control group did not receive concept mapping instruction or practice and was not required to provide evidence of using metacognitive strategies with graphic organizers in preparation for course examinations. However, like students in the treatment groups, students in the control group were required to contact their instructors on a weekly basis via e-mail or telephone to ask questions about course material and concepts that they did not understand from any of the lectures, reading assignments, or problemsolving assignments.

The course learning objectives did not change over the course of this study. Every effort was made to ensure that all aspects of the course, such as learning objectives-driven lectures, laboratories, problem-solving and homework assignments, question-answer sessions, weekly e-mail correspondence, and examinations, were equivalent for the non-concept mapping control group and the concept mapping treatment group.

\section{Instrumentation}

The outcomes measures used for the comparative analysis were based on the final examination for the radiation protection and radiobiology course. The comprehensive final examination consists of 100 questions of various levels of difficulty, from knowledge-based memorization of definitions and regulations to synthesis- and evaluationlevel questions relating to problem-solving situations that a technologist may encounter during a workday. These situations include shielded and unshielded exposure calculations, inverse square law, and radioactive decay leading to technologist or patient dose calculations.

\section{RESULTS}

The mean score on the introductory radiation protection and radiobiology final examination for the distance learning students who used concept mapping as a learning strategy was 72.3 (SE, 3.45); the median score was 70. The mean score for the distance learning students who did not use concept mapping as a learning strategy was 57.3 (SE, 5.67); the median score was 57 . The effect size-or actual, meaningful differences in the mean scores-was 2.65; this value is considered to represent a very large effect size (14). The Mann-Whitney $U$ test for differences in the median scores indicated a significant difference in outcomes $(z=$ $-2.0381, P=0.0415)$. This difference is visually depicted by the displacement of the box plots in Figure 2 .

\section{DISCUSSION}

The quantitative analysis supported the use of concept mapping as a metacognitive strategy or learning intervention by students in the distance learning nuclear medicine technology program. The difference in median scores was statistically significant, and the effect size was very large. The literature suggests that using concept maps encouraged students to think about what they were learning instead of engaging in rote memorization. Additionally, concept maps allowed for directed remediation when needed, furthering understanding without misconceptions. This deeper understanding of the relationships between concepts enabled students who used concept mapping to be better problem solvers, as exhibited in the final examination scores.

Figure 3 represents a student concept map of fundamental radiation and radiation decay concepts created without the meaningful learning advantage gained by considering what the relationships between the concepts mean. This representation is essentially an organizational chart in which some sense of hierarchy is achieved by placement or position but in which the real relationships between the 


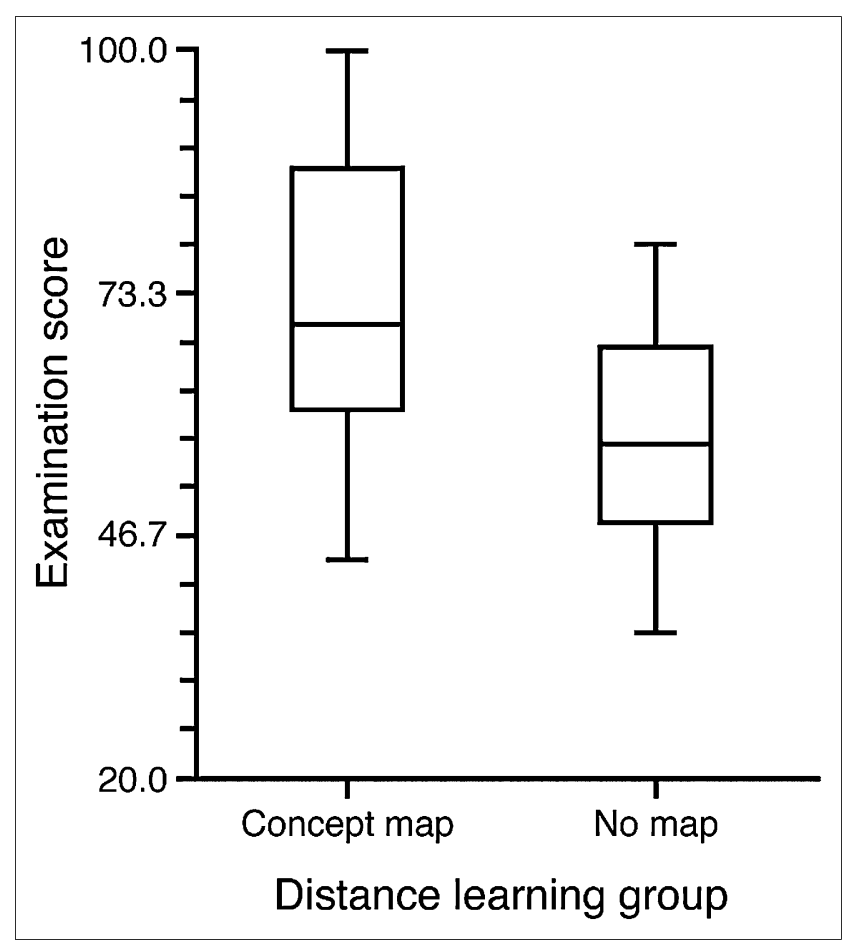

FIGURE 2. Box plots depicting differences in scores between concept mapping group (treatment group) and non-concept mapping group (control group; no map). Median score for treatment group was significantly higher than median score for control group $(z=-2.0381 ; P=0.0415)$.

concepts are not expressed. The student confused the concept of "activity" with the concept of "intensity" in terms of the use of distance and shielding as protective measures. Anecdotally, this student did not score as high as the student whose concept map is represented by Figure 1; this student's score actually was closer to the median score for the non-concept mapping group than it was to the median score for the concept mapping group. Furthermore, this student expressed dissatisfaction with the concept mapping procedure, claiming that it was too time-consuming and therefore interfered with learning (memorization) techniques. Remediation in this case was limited to identification or clarification of gross errors, with little remediation of misconceptions for understanding, because the map itself was the template for the remedial communication exchange.

As a recommendation for further research, given the anecdotal evidence described here, it would be interesting to investigate the learning effectiveness of concept mapping versus other graphic organizers or formative assessment techniques. Such an investigation would help determine whether the learning gains noted in this study were the result of the learning intervention or some other study method used by the treatment group and not accounted for. Alternatively, using learning interventions without the remedial aspects involved in checking for comprehension could be attempted; however, students need and expect feedback before assessments, and it is conceptually difficult

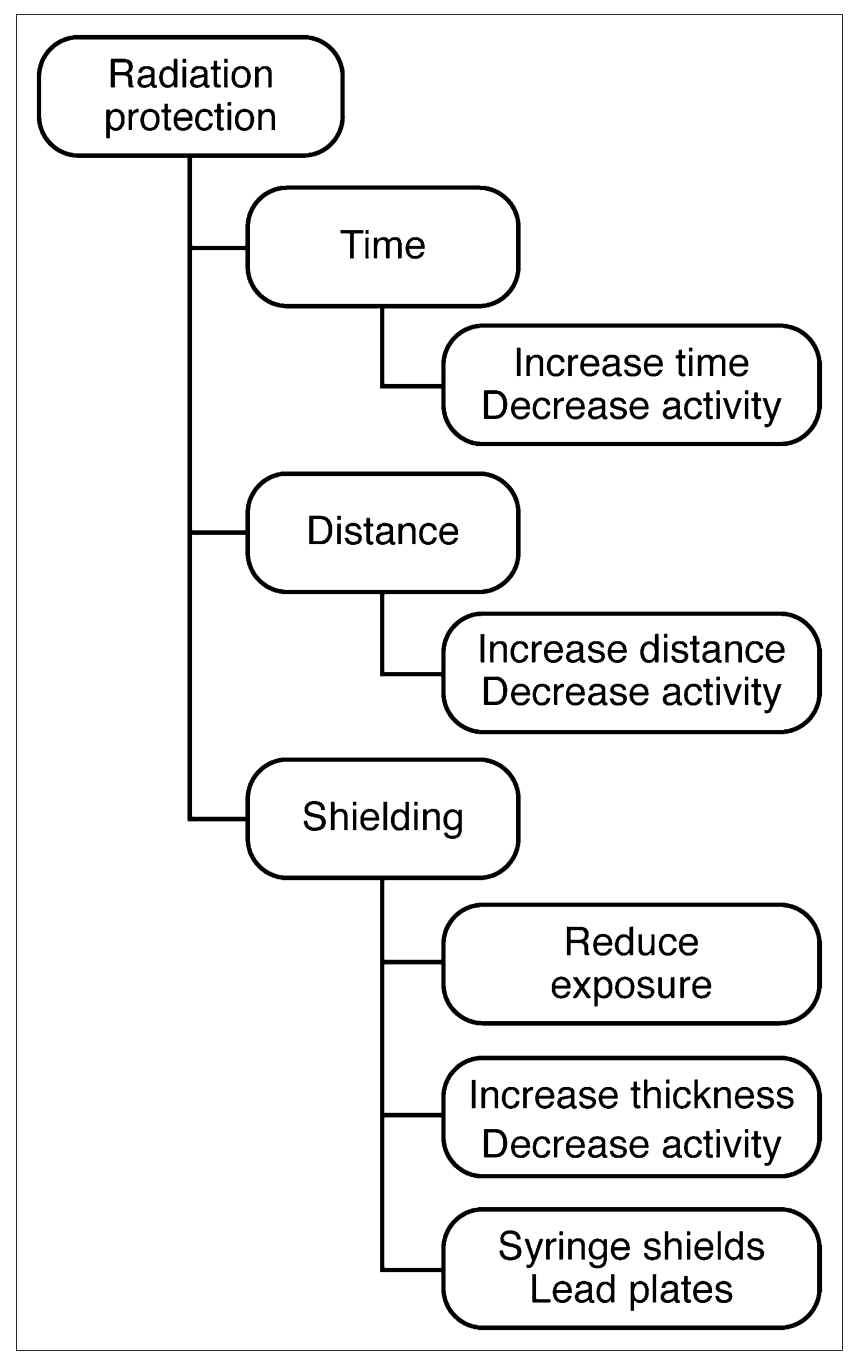

FIGURE 3. Student "concept map" based more on organizational chart structure than on concept map strategy that identifies relationships between concepts. Student confused concept of "activity" with concept of "radiation intensity" in terms of use of distance and shielding as protective measures. This student's "concept map" was used to guide attempts at misconception remediation because this student was experiencing conceptualization difficulties.

to envision how such a comparison could be implemented in an academic environment.

\section{CONCLUSION}

This study confirmed that concept mapping is a cognitive learning intervention and remediation tool that effectively enables meaningful learning. Furthermore, this study indicated that concept mapping is suitable for use in the independent learner-oriented distance learning environments used by some nuclear medicine technology programs.

\section{ACKNOWLEDGMENT}

No potential conflict of interest relevant to this article was reported. 


\section{REFERENCES}

1. Ertmer PA, Newby TJ. Behaviorism, cognitivism, constructivism: comparing critical features from an instructional design perspective. Performance Improvement Quarterly. 1993;6:50-72.

2. Gonzalez C. Conceptions of, and approaches to, teaching online: a study of lecturers teaching postgraduate distance courses. Higher Education. 2009;57:299-314.

3. Kember D, Kwan K-P. Lecturers' approaches to teaching and their relationship to conceptions of good teaching. Instructional Science. 2000;28:469-490.

4. Novak JD, Cañas AJ. The theory underlying concept maps and how to construct and use them. Technical report IHMC CmapTools 2006-01 Rev 01-2008. Florida Institute for Human and Machine Cognition. Available at: http://cmap.ihmc.us/ Publications/ResearchPapers/TheoryUnderlyingConceptMaps.pdf. Revised January 22, 2008. Accessed October 20, 2011.

5. Passmore GG. The Effects of Gowin's Vee Heuristic Diagramming and Concept Mapping on Meaningful Learning in the Radiation Science Classroom and Laboratory [dissertation]. Columbia, MO: University of Missouri; 1996.

6. Novak JD, Gowin DB. Learning How to Learn. New York, NY: Cambridge University Press; 1984.

7. Passmore GG. Constructing concept maps facilitates learning in radiologic technologies education. Radiologic Science and Education. 1995;2:50-59.
8. Passmore GG. Using Vee diagrams to facilitate meaningful learning and misconception remediation in radiologic technologies laboratory education. Radiologic Science and Education. 1998;4:11-28.

9. Dansereau DF, Cross DR. Knowledge Mapping: Cognitive Software for Thinking, Learning, and Communicating. Ft. Worth, TX: Texas Christian University Publications; 1990.

10. Angelo TA, Cross KP. Classroom Assessment Techniques: A Handbook for College Teachers. 2nd ed. San Francisco, CA: Jossey-Bass; 1993.

11. Novak JD. Concept mapping: a useful tool for science education. J Research Sci Teaching. 1990;27:937-949.

12. Heinze-Fry JA, Novak JD. Concept mapping brings long-term movement toward meaningful learning. Sci Educ. 1990;74:461-472.

13. Canas AJ, Coffey JW, Carnot MJ, Feltovich P, Hoffman RR, Novak JD. A summary of literature pertaining to the use of concept mapping techniques and technologies for education and performance support. Florida Institute for Human and Machine Cognition, Pensacola, FL. Available at: http://www.ihmc.us/users/acanas/ Publications/ConceptMapLitReview/IHMC\%20Literature\%20Review\%20on\% 20Concept\%20Mapping.pdf. Published 2003. Accessed February 24, 2009.

14. Cohen J. Statistical Power for the Behavioral Sciences. 2nd ed. Hillsdale, NJ: Lawrence Erlbaum Associates; 1988. 